\title{
INFLUÊNCIA DO DIÂMETRO E UMIDADE NO TRATAMENTO PRESERVATIVO DE MOIRÕES DE Eucalyptus ${ }^{1}$
}

\author{
Lucas Soares Amaral², José Reinaldo Moreira da Silva ${ }^{3}$, Paulo Ricardo Gherardi Hein ${ }^{3}$ e Paulo Fernando \\ Trugilho $^{3}$
}

\begin{abstract}
RESUMO - Objetivou-se avaliar os efeitos da classe diamétrica e das umidades geral e do alburno sobre a retenção de CCA-C (arseniato de cobre cromatado tipo C) na madeira de clone de Eucalyptus urophylla preservada em autoclave. Foram utilizados 36 moirões pertencentes às classes diamétricas de 7, 9, 11 e $13 \mathrm{~cm}$, que foram separados e expostos a três tempos de secagem ao ar livre: 20, 40 e 70 dias. Determinaram-se os valores de umidade pré-tratamento, geral e apenas do alburno, por classe diamétrica e por tempo de secagem. Após a preservação em autoclave, foi feito o controle de qualidade do tratamento preservativo, por meio da análise de retenção do CCA-C. A retenção nos moirões da classe de $7 \mathrm{~cm}$ foi superior à das classes de 9, 11 e 13 $\mathrm{cm}$, que não variaram estatisticamente entre si. O aumento do tempo de secagem de 20 para 40 dias proporcionou incremento significativo na retenção média de 5,70 para 6,67 kg I.A* $\mathrm{m}^{-3}$. As variações médias das umidades geral e do alburno se mostraram significativas com a variação da retenção, apresentando boa correlação entre os fatores. A correlação entre a umidade geral e a retenção de CCA-C $(r=-0,86)$ foi mais forte que a correlação entre a umidade do alburno e a retenção $(r=-0,70)$.
\end{abstract}

Palavras-chave: Preservação da madeira; Qualidade; CCA-C.

\section{INFLUENCE OF DIAMETER AND MOISTURE ON PRESERVATIVE TREATMENT OF EUCALYPTUS FENCE POSTS}

\begin{abstract}
The aim of this study was to assess the effects of diameter class, general moisture and sapwood moisture over the retention of CCA-C (Chromated Copper Arsenate type C) on a Eucalyptus urophylla clone wood preserved in autoclave. 36 fence posts were used, belonging to the diameter classes of 7, 9, 11 and $13 \mathrm{~cm}$, which were separated and exposed to three outdoor drying times: 20, 40 and 70 days. The pre-treatment moisture values, general and only sapwood, were determined by diameter class and drying times. After the preservation in autoclave, the treatment quality control was made by analyzing the CCA-C retention. Retention in fence posts of the $7 \mathrm{~cm}$ class was higher than those of 9, 11 and $13 \mathrm{~cm}$ classes, which did not vary statistically amongst each other. The increase in drying time from 20 to 40 days resulted in significant increase in average retention from 5.70 to $6.67 \mathrm{~kg}$ A.I. $*^{*} \mathrm{~m}^{-3}$. The mean variations of the general moisture and the sapwood were significant with variation of retention, showing good correlation between the factors. The correlation between the fence posts general moisture and retention of CCA-C $(r=-0.86)$ was stronger than the correlation between sapwood moisture and retention $(r=-0.70)$.
\end{abstract}

Keywords: Wood preservation; Quality; CCA-C.

\footnotetext{
${ }^{1}$ Recebido em 07.10.2012 aceito para publicação em 07.08.2014.

${ }^{2}$ Programa de Pós Graduação em Ciências Florestais, Universidade Federal de Lavras, MG, Brasil. E-mail: <amaralslucas@hotmail.com>. ${ }^{3}$ Departamento de Ciências Florestais, Universidade Federal de Lavras, MG Brasil.E-mail: <jreinaldo@dcf.ufla.br>, <paulohein@dcf.ufla.br> e<trugilho@dcf.ufla.br>.
} 


\section{INTRODUÇÃO}

No Brasil, a madeira de Eucalyptus é utilizada em grande quantidade nas indústrias de preservação, principalmente na forma roliça. De acordo com Jankowsky et al. (2002), a preservação de madeiras é entendida, usualmente, como a aplicação de produtos químicos, visando impedir a degradação física e química ou, principalmente, a deterioração biológica do material madeira.

Para comercialização de seus produtos, as empresas produtoras de madeira tratada devem atender a normas e padrões de qualidade, as quais incluem a penetração e retenção do produto preservativo. Segundo Hunt e Garratt (1967), a retenção representa a quantidade de preservativo contida em determinado volume de madeira, sendo o fator mais importante no desempenho do material tratado. Valores mínimos de retenção são exigidos de acordo com o uso e grau de exposição das peças. Para o uso de moirões em contato com o solo, a NBR 9480 (ABNT, 2009) determina que os valores não podem ser inferiores a $6,5 \mathrm{~kg}$ I.A. ${ }^{*} \mathrm{~m}^{-3}$.

Mendes e Alves (1988) descreveram que os métodos de tratamento com pressão garantem a qualidade de tratamento superior, com melhor proteção da madeira. Por isso, a NBR 9480 (ABNT, 2009) recomenda que a preservação da madeira de moirões seja realizada somente pelo processo de vácuo-pressão.

Para atingir os valores mínimos estabelecidos, as características do processo exercem influência direta, porém as características da madeira também devem ser levadas em consideração. Para uma usina de preservação de madeiras, em que se utiliza apenas um material genético selecionado, há duas fontes principais de variação na madeira: diâmetro e umidade.

A variação do diâmetro em madeiras de mesmo material genético corresponde à variação de idade e posição longitudinal no tronco, o que pode implicar variações de características anatômicas, físicas, mecânicas e químicas e, consequentemente, afetar a qualidade do tratamento preservativo.

Segundo Lepage (1986) e Santini (1988), a umidade da madeira tem forte influência na qualidade do tratamento preservativo. Para processos de tratamento em autoclave, aumentos da umidade da madeira acima do ponto de saturação das fibras diminuem a fração de vazios, podendo impedir a retenção desejada de preservativo. A umidade da madeira para que a penetração e retenção do preservativo sejam satisfatórias varia com o método de tratamento e o tipo de preservativo utilizado. Para processos industriais com pressão, é recomendado o uso de madeira com umidade abaixo do PSF. Assim, Usta (2004) informou que a necessidade da secagem antes do tratamento sob vácuo-pressão é bem conhecida, mas ainda não se conhecem os valores ótimos de umidade para as espécies de madeira. Outro fato não informado é a importância de se trabalhar com a umidade média geral das peças ou com apenas a umidade do alburno, que corresponde à região tratável da madeira de Eucalyptus.

Assim, objetivou-se avaliar os efeitos da classe diamétrica e das umidades geral e do alburno na retenção de CCA-C (arseniato de cobre cromatado tipo C) na madeira de Eucalyptus preservada em autoclave.

\section{MATERIAL E MÉTODOS}

A madeira foi proveniente de povoamento clonal de Eucalyptus urophylla, da empresa Plantar S. A., com 9 anos de idade, plantado em espaçamento 3 x 3 m e localizado no Município de Felixlândia, MG. Durante o processo de colheita da empresa, de forma aleatória foram coletadas e descascadas 36 toras de 3,00 m, pertencentes às classes diamétricas de 6,0 a 7,9; 8,0 a 9,9; 10,0 a 11,9; e 12,0 a 13,9 cm, que foram expostas à secagem ao ar livre por diferentes períodos de tempo, conforme descrito na Tabela 1.

Ao final de cada tempo de secagem, para eliminar a influência da secagem de topo, descartaram-se toretes de $35 \mathrm{~cm}$ em cada extremidade das toras, e retirou-se um disco de $5 \mathrm{~cm}$ para determinação da umidade, conforme Norma NBR 7190 (ABNT, 1997). A partir dos discos, produziram-se corpos de prova de $2 \times 2 \times 5 \mathrm{~cm}$, formando uma malha quadriculada, cujas posições foram identificadas e, posteriormente, determinada a umidade de cada corpo de prova. Assim, foi possível conhecer os valores de umidade média geral e a umidade do alburno, em cada classe de diâmetro.

Após a amostragem para determinação da umidade, os moirões foram submetidos ao tratamento preservativo industrial. As toras foram inseridas nas bateladas habituais da Empresa Plantar Empreendimentos e Produtos Florestais Ltda., e o processo consistiu em: $1^{\circ}$ ) Carregamento da autoclave; $2^{\circ}$ ) Vácuo inicial de 
Tabela 1 - Número de moirões por classe diamétrica e por tempo de secagem no campo. Table 1 - Fence posts number by diameter class and drying time in the field.

\begin{tabular}{|c|c|c|c|c|c|}
\hline \multirow[t]{2}{*}{ Tempo de secagem (dias) } & \multicolumn{4}{|c|}{ Centro da classe diamétrica $(\mathrm{cm})$} & \multirow[t]{2}{*}{ Total } \\
\hline & 7 & 9 & 11 & 13 & \\
\hline 20 & 3 & 4 & 3 & 2 & 12 \\
\hline 40 & 3 & 4 & 2 & 3 & 12 \\
\hline 70 & 3 & 3 & 3 & 3 & 12 \\
\hline Total & 9 & 11 & 8 & 8 & 36 \\
\hline
\end{tabular}

550 mmHg, durante 30 min; $3^{\circ}$ ) Injeção do preservativo sob pressão $\left(12 \mathrm{kgf} / \mathrm{cm}^{2}\right)$ durante $60 \mathrm{~min}$; e $4^{\circ}$ ) vácuo final de $550 \mathrm{mmHg}$, durante $15 \mathrm{~min}$.

O produto preservativo utilizado foi o CCA $60 \%$ tipo C (Osmose K33 C 60, da Montana Química S. A.). Nos tratamentos após 20 e 40 dias de secagem, utilizou-se a concentração da solução de $1,7 \%$, enquanto no tratamento após 70 dias de secagem a concentração teve seu valor alterado para 1,5\%, devido a fatores operacionais da empresa. Essa variação na concentração não foi objetivo de avaliação deste trabalho.

Após o tratamento, as toras foram transportadas para o pátio de estocagem ao ar livre. Vinte dias após o último tratamento, período suficiente para a ocorrência das reações de fixação do preservante, foi realizada a amostragem para a análise de retenção, por meio da Espectrometria de raios $\mathrm{X}$, conforme os padrões estabelecidos na Norma NBR 6232 (ABNT, 2013).

Utilizando furadeira e broca extratora, foram retiradas cinco baguetas por tora, em posições aleatórias, distantes no mínimo $50 \mathrm{~cm}$ do topo e da base. As baguetas apresentavam formato cilíndrico, com comprimento igual à espessura do alburno. Foram medidos o comprimento e os diâmetros. Calculando o volume, após a secagem em estufa por $3 \mathrm{~h}$ a $[103 \pm 2]^{\circ} \mathrm{C}$, determinou-se a massa de cada bagueta. A partir dos volumes e das massas secas, foi calculada a densidade aparente $\left(\mathrm{g}^{*} \mathrm{~cm}^{-3}\right)$, com a média dos valores das cinco baguetas consideradas como a densidade aparente do moirão tratado.

Na empresa Montana Química S. A., foi feita a moagem das baguetas em moinho tipo Willye, modelo TE-680. O material com granulometria inferior a 30 mesh foi coletado e identificado, por moirão. Esse material foi transferido para cubetas plásticas montadas com filme Mylar para raios X. Para determinação dos ingredientes ativos, utilizou-se o Espectrômetro de raios X Shimadzu - EDX-720, e a retenção foi calculada conforme a equação 1 .

$$
\mathrm{R}=\mathrm{LE} * \rho * 10
$$

em que:

$$
\mathrm{R} \text { = retenção, em kg I.A* } \mathrm{m}^{-3} \text {; }
$$

LE = leitura obtida no equipamento; e

$\rho=$ densidade aparente média, em $g^{*} \mathrm{~m}^{-3}$.

Os cálculos foram feitos para os componentes do CCA, individualmente: cromo, cobre e arsênio. Assim, a retenção total foi igual à somatória das retenções individuais.

A coleta de dados obedeceu ao Delineamento Inteiramente Casualizado (DIC), disposto em arranjo fatorial. Dessa forma, utilizou-se a análise de variância a 5\% de significância, considerando-se os efeitos de classe diamétrica, tempo de secagem e interação entre esses fatores. Quando a análise foi significativa, aplicou-se o teste de Tukey a 5\% de significância. Foram avaliadas também as correlações entre as características da madeira e a retenção de CCA-C. Para tanto, utilizaram-se o coeficiente de correlação de Pearson e o teste t, de Student, a 5\% de significância.

\section{RESULTADOS}

A variação da umidade geral dos moirões foi significativa entre classes diamétricas e entre tempos de secagem. Já a umidade do alburno diferiu estatisticamente apenas entre os tempos de secagem. Houve diferença significativa entre a retenção de CCAC nos moirões de diferentes classes diamétricas, independentemente do tempo de secagem e entre os tempos de secagem a que foram submetidos os moirões, independentemente da classe diamétrica. A interação classe $\mathrm{x}$ tempo não foi significativa para nenhuma das características (Tabela 2).

Os valores médios de umidade foram 29,16\% para umidade geral, com máximo de 55,43\% e mínimo de 10,98\%; e 21,46\% para a umidade do alburno, com máximo de 36,62\% e mínimo de 10,98\%.

Revista Árvore, Viçosa-MG, v.38, n.5, p.919-925, 2014 
Tabela 2 - Resumo da ANAVA para umidade geral, umidade do alburno e retenção de CCA-C em moirões de um clone de Eucalyptus urophylla.

Table 2 - Summary of ANOVA for general moisture, sapwood moisture and CCA-C retention in Eucalyptus urophylla clone fence posts.

\begin{tabular}{|c|c|c|c|c|c|c|}
\hline \multirow[t]{2}{*}{ Fonte de variação } & \multicolumn{2}{|c|}{ Umidade geral } & \multicolumn{2}{|c|}{ Umidade do alburno } & \multicolumn{2}{|c|}{ Retenção } \\
\hline & GL & QM & GL & QM & GL & QM \\
\hline Classe diamétrica & 3 & $727,78 *$ & 3 & 19,98 & 3 & $1,159 *$ \\
\hline Tempo de secagem & 2 & $1450,51 *$ & 2 & $830,45^{*}$ & 2 & $6,128 *$ \\
\hline Classe x Tempo & 6 & 20,47 & 6 & 10,47 & 6 & 0,499 \\
\hline Resíduo & 24 & 9,13 & 24 & 6,70 & 24 & 0,215 \\
\hline Total & 35 & & 35 & & 35 & \\
\hline
\end{tabular}

* = significativo a 5\% de significância.

* = significant at $5 \%$ significance.

As comparações múltiplas entre classes diamétricas e entre tempos de secagem, para os valores de umidade geral dos moirões e umidade do alburno destes, são apresentadas nas Tabelas 3 e 4.

A retenção média de CCA-C foi de 5,88 kg I.A*m³, com um máximo de 7,93 kg I.A*m³ e mínimo de 4,16 $\mathrm{kg} I . \mathrm{A}^{*} \mathrm{~m}^{-3}$.

A distribuição da retenção média de CCA-C dos moirões, por classe diamétrica e após os três tempos de secagem, encontra-se na Figura 1.

Para o estudo das correlações, consideraram-se apenas os dados referentes aos moirões com tempos de secagem de 20 e 40 dias. Os valores referentes aos moirões com 70 dias de secagem não foram considerados devido à influência da diminuição da concentração da solução na retenção, conforme citado anteriormente.

Tabela 3 - Comparações múltiplas das médias de umidade geral e umidade do alburno dos moirões, por classe diamétrica.

Table 3 - Multiple comparisons of the means of fence posts general moisture and sapwood moisture, by diameter class.

\begin{tabular}{|c|c|c|}
\hline \multirow{2}{*}{$\begin{array}{c}\text { Classe diamétrica } \\
(\mathrm{cm})\end{array}$} & \multicolumn{2}{|c|}{ Umidade (\%) } \\
\hline & Geral & Alburno \\
\hline 7 & 19,24 a $(46,83)$ & $19,24 \mathrm{~ns}(46,83)$ \\
\hline 9 & 25,53 b $(32,33)$ & $22,60(38,84)$ \\
\hline 11 & 33,87 с $(36,09)$ & $21,55(28,64)$ \\
\hline 13 & 40,45 d $(26,78)$ & $21,93(28,78)$ \\
\hline
\end{tabular}

Médias seguidas de pelo menos uma mesma letra na coluna não diferem entre si, pelo teste de Tukey a 5\% de significância. Valores entre parênteses correspondem ao coeficiente de variação, em \%. ns = não significativo, pelo teste de Tukey a $5 \%$ de significância. Means followed by the same letter in the column does not differ amongst each other, by Tukey test at 5\% significance. Values in parentheses correspond to the coefficient of variation, in \%. $n \mathrm{~s}=$ non-significant, by Tukey test at the 5\% significance.

Revista Árvore, Viçosa-MG, v.38, n.5, p.919-925, 2014
Tabela 4-Comparações múltiplas das médias de umidade geral eumidade do alburno dos moirões, por tempo de secagem

Table 4-Multiple comparisons of the means of fence posts general moisture and sapwood moisture, by drying time.

\begin{tabular}{|c|c|c|}
\hline \multirow{2}{*}{$\begin{array}{c}\text { Tempo de secagem } \\
\text { (dias) }\end{array}$} & \multicolumn{2}{|c|}{ Umidade (\%) } \\
\hline & Geral & Alburno \\
\hline 20 & 42,11 a $(24,12)$ & 31,00 a $(10,94)$ \\
\hline 40 & 26,99 b $(33,83)$ & 17,97 b $(16,96)$ \\
\hline 70 & 20,22 с $(36,78)$ & 15,02 с $(15,32)$ \\
\hline
\end{tabular}

Médias seguidas de pelo menos uma mesma letra na coluna não diferem entre si, pelo teste de Tukey a 5\% de significância. Valores entre parênteses correspondem ao coeficiente de variação, em \%.

Means followed by the same letter in the column does not differ amongst each other, by Tukey test at 5\% significance. Values in parentheses correspond to the coefficient of variation, in $\%$

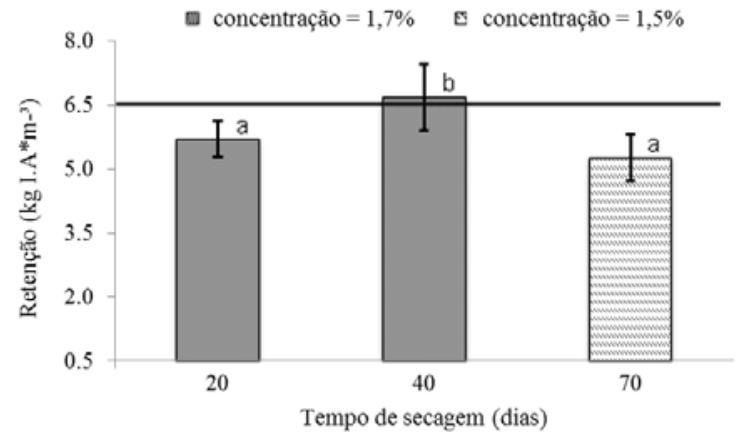

Figura 1 - Retenção média de CCA-C por classe diamétrica (A) e por tempo de secagem (B), com os respectivos desvios-padrão.

Figure 1 - Average retention of CCA-C by diameter class (A) and drying time (B), with the respective standard deviations.

A retenção de CCA-C apresentou correlações significativa e negativa nas três características da madeira analisadas: umidade geral, umidade do alburno e diâmetro dos moirões; com coeficientes de correlação (r) de 0,86; -0,70; e -0,52, respectivamente. 


\section{DISCUSSÃO}

Comparando as umidades após cada tempo de secagem, todas diferiram estatisticamente entre si, com tendência natural de aproximação dos valores da umidade geral com a umidade do alburno, à medida que se aumentou o tempo de secagem. Apesar de a média geral mostrar que, com 40 dias de secagem ao ar livre, os moirões apresentavam umidade inferior a 30\%, analisando-se os dados individualmente, somente após 70 dias de secagem os moirões da classe de $13 \mathrm{~cm}$ atingiram esse ponto, ou seja, de acordo com a literatura, estavam aptos para o tratamento preservativo em autoclave.

A variação de umidade encontrada está de acordo com o exposto por Oliveira et al. (2005), que, trabalhando com árvores recém-abatidas, afirmaram que a espécie Eucalyptus urophylla apresenta alta variabilidade quanto à distribuição radial da umidade. Esses autores chegaram à queda de umidade no sentido medula-casca, com diferença de $80 \%$ da região mais interna para a mais externa do tronco. Também, demonstraram que a umidade do alburno da tora será menor que a do cerne e, consequentemente, menor que a umidade média geral da madeira. Neste trabalho, essa situação se manteve, mesmo após 70 dias de secagem ao ar livre, o que demonstra que, com o passar do tempo, a madeira tende a entrar em equilíbrio com a umidade relativa e a temperatura ambiente, porém com a existência de gradiente em seu interior.

Comparando as retenções entre classes diamétricas, os valores foram iguais estatisticamente entre os moirões das classes de 9,11 e $13 \mathrm{~cm}$, que por sua vez foram diferentes daqueles pertencentes à classe de 7 cm.

Os moirões das classes de 9, 11 e 13 cm apresentaram retenção média de 5,70 kg I.A* $\mathrm{m}^{-3}$, contra 6,41 kg I.A*m³ dos moirões da classe de $7 \mathrm{~cm}$. Em termos médios, os valores de retenção ficaram abaixo do mínimo estabelecido pela NBR 9480 (ABNT, 2009), que é de $6,5 \mathrm{~kg} \mathrm{I.A*} \mathrm{m}^{-3}$. Porém, seis peças (17\% do total), distribuídas entre as classes de 7, 9 e 11 cm, apresentaram retenções acima do estabelecido.

Houve diferença significativa entre as retenções de CCA-C em moirões com 20 e 40 dias de secagem. Os moirões com 70 dias de secagem não mantiveram a tendência de aumento na retenção, apresentando valores estatisticamente iguais aos daqueles com 20 dias. Porém, conforme já mencionado e exposto na Figura 2, a diminuição da retenção foi influenciada pela diminuição na concentração da solução de tratamento, não sendo possível determinar o efeito da secagem na retenção apresentada por essas peças. Evangelista (2011) demonstrou que alterações de $0,5 \%$ na concentração da solução de tratamento, para 60 min de pressão na autoclave, podem acarretar diferenças de até $29 \%$ na retenção do CCA-C.

As maiores retenções apresentadas pelas peças com 40 dias de secagem estão em conformidade com a umidade média das peças obtida após esse período, ou seja, menor que $30 \%$. Tal fato corroborou a literatura em geral, segundo a qual, para melhores resultados no tratamento em autoclave, a madeira deve apresentar umidade abaixo do PSF.

Com 20 dias de secagem, nenhum moirão atingiu o mínimo de retenção estabelecido pela NBR 9480 (ABNT, 2009). Já com 40 dias, embora na média geral os moirões tenham atingido a retenção mínima necessária, novamente seis moirões (17\% do total) apresentaram valores inferiores, com a ressalva de que, entre eles, estavam todos da classe de $13 \mathrm{~cm}$.

Analisando o coeficiente de correlação entre o diâmetro dos moirões e a retenção de CCA-C nestes, trata-se de correlação moderada, devido à influência da umidade dessas peças. Já as correlações entre a retenção e a umidade da madeira foram fortes.

Analisando o coeficiente de determinação $\left(\mathrm{r}^{2}\right)$, pode-se afirmar que $74 \%$ da variação total da retenção de CCA-C pode ser explicada pela variação da umidade média geral da madeira. Considerando apenas a umidade do alburno, o coeficiente de determinação demonstra que $49 \%$ da variação da retenção de CCA-C pode ser explicada pela variação da umidade do alburno. Esses resultados indicam que a existência de elevado gradiente de umidade no interior das peças parece influenciar a retenção de CCA-C, de modo que, para melhor controle do tratamento preservativo, é importante levar em consideração não somente a umidade do alburno, mas a umidade geral das peças.

Valle (2009) e Evangelista (2011) avaliaram correlações de retenção de CCA com diversas propriedades da madeira de Eucalyptus, concluindo que as propriedades da madeira não contribuíram para explicar os resultados de retenção. Porém, nesses trabalhos a umidade utilizada foi abaixo do PSF, não 
sendo determinada e analisada a umidade da madeira pré-tratamento. O efeito da umidade na retenção de CCA foi mais estudado em madeiras de coníferas. Assim, Kumar e Morrell (1989) concluíram que o tratamento de peças com 18\% de umidade apresentaram melhores resultados do que aquelas com $28 \%$, resultado semelhante ao encontrado neste trabalho, considerando-se a umidade do alburno. Esses autores afirmaram ainda que não foi considerada vantajosa a secagem até $9 \%$ de umidade. Esses resultados também estão de acordo com os encontrados por Usta (2004), quando verificou que valores abaixo do PSF proporcionaram maior absorção do produto, sendo a maior absorção obtida na umidade de $22 \%$, e a secagem, além desse ponto, não representa melhoria na qualidade do tratamento e parece ser inviável economicamente.

\section{CONCLUSÕES}

1)A retenção nos moirões da classe de $7 \mathrm{~cm}$ foi superior à das classes de 9 cm, 11 cm e 13 cm, que não variaram entre si.

2)O aumento do tempo de secagem de 20 para 40 dias proporcionou incremento significativo na retenção média de CCA-C, de 5,70 para 6,67 kg I.A.* ${ }^{*}{ }^{-3}$.

3)A variação do diâmetro dos moirões se mostrou significativa com a variação da retenção, com correlação moderada $(r=-0,52)$.

4)As variações da umidade geral e do alburno se mostraram significativas com a variação da retenção, com boa correlação entre os fatores.

5)A correlação entre a umidade geral dos moirões e a retenção de CCA-C $(r=-0,86)$ foi mais forte que a correlação entre a umidade do alburno e a retenção $(\mathrm{r}=-0,70)$.

\section{AGRADECIMENTOS}

À Coordenação de Aperfeiçoamento de Pessoal de Nível Superior (CAPES), pelo apoio; à Fundação de Amparo à Pesquisa do Estado de Minas Gerais (FAPEMIG), por conceder recursos financeiros para bolsas de estudo e aquisição de suplementos, respectivamente; à Plantar Empreendimentos e Produtos Florestais Ltda. e à Montana Química S. A., pelo apoio logístico na coleta de dados, que foram fundamentais para a realização deste trabalho.

Revista Árvore, Viçosa-MG, v.38, n.5, p.919-925, 2014

\section{REFERÊNCIAS}

ASSOCIAÇÃO BRASILEIRA DE NORMAS TÉCNICAS - ABNT. NBR 6232: penetração e retenção de preservativos em madeira tratada sob pressão. Rio de Janeiro: 2013. 16p.

ASSOCIAÇÃO BRASILEIRA DE NORMAS TÉCNICAS - ABNT._ NBR 7190: projeto de estruturas de madeira. Rio de Janeiro: 1997. 107 p.

ASSOCIAÇÃO BRASILEIRA DE NORMAS TÉCNICAS - ABNT._NBR 9480: peças roliças preservadas de eucalipto para construções rurais - Requisitos. Rio de Janeiro: 2009. 12p.

EVAngelista, W. V. Penetração e retenção de arseniato de cobre cromatado em madeira de eucalipto. 2011. 105f. Tese (Doutorado em Ciência Florestal) - Universidade Federal de Viçosa, Viçosa, MG, 2011.

HUNT, G. M.; GARRATT, G. A. Wood preservation. 3.ed. New York: McGraw Hill, 1967. 433p.

JANKOWSKY, I. P.; BARILLARI, C. T.; FREITAS, V. P. A preservação de madeiras no Brasil.

Revista da Madeira, v.67, n.1, p.49-50, 2002.

KUMAR, S.; MORRELL, J. J. Moisture content of western hemlock: influence on treatability with chromated copper arsenate Type C.

Holzforschung, v.43, n.4, p.279-280, 1989.

LEPAGE, E. S. Manual de preservação de madeiras. São Paulo: IPT, 1986. p.523-540.

MENDES, A. S.; ALVES, M. V. S. A

degradação da madeira e sua preservação. Brasília: IBDF/DPq-LPF, 1988. $56 \mathrm{p}$.

OLIVEIRA, J. T. S.; HELLMEISTER, J. C.; TOMAZELLO FILHO, M. Variação do teor de umidade e da densidade básica na madeira de sete espécies de eucalipto. Revista Árvore, v.29, n.1, p.115-127, 2005.

USTA, I. The effect of moisture content and wood density on the preservative uptake of Caucasian fir (Abiesnordmanniana 
(Link.)Spach.)treated with CCA. Turkish Journal of Agriculture and Forestry, v.28, n.1, p.1-7, 2004.

SANTINI, E. J. Biodeterioração e preservação da madeira. Santa Maria: UFSM/CEPEF/FATEC, 1988. 125 p.
VAlle, M. L. A. Propriedades da madeira de eucalipto de primeira e segunda rotação, visando a sua utilização como madeira preservada. 2009. 80f. Dissertação (Mestrado em Ciência Florestal) - Universidade Federal de Viçosa, Viçosa, MG, 2009. 